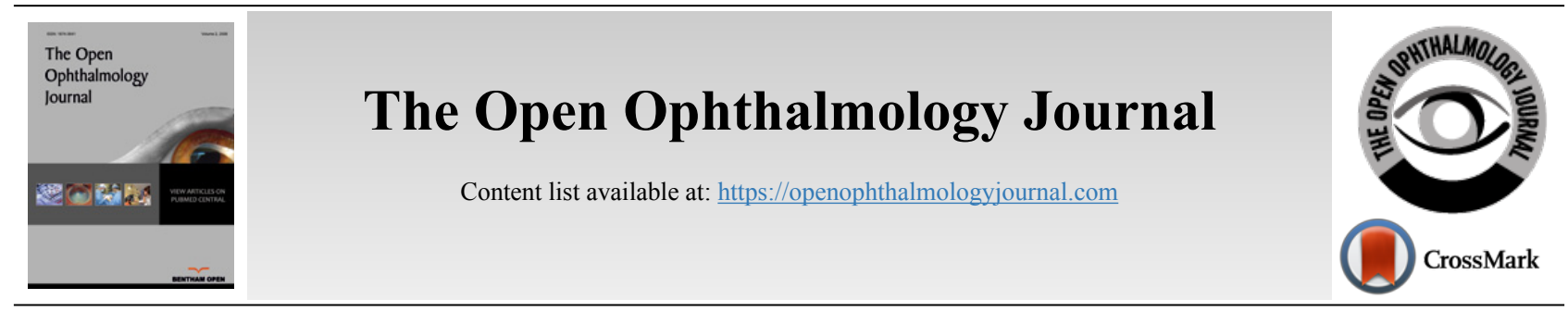

RESEARCH ARTICLE

\title{
Silent Information Regulator T1 in Aqueous Humor of Patients with Age- Related Macular Degeneration
}

\author{
Tatsuya Mimura ${ }^{1,2, *}$, Hideharu Funatsu ${ }^{3}$, Hidetaka Noma $^{4}$, Aki Kondo $^{2}$ and Atsushi Mizota ${ }^{1}$ \\ ${ }^{1}$ Department of Ophthalmology, School of Medicine, Teikyo University, Tokyo, Japan \\ ${ }^{2}$ Department of Ophthalmology, Tokyo Women's Medical University Medical Center East, Tokyo, Japan \\ ${ }^{3}$ Department of Ophthalmology, Yachiyo Medical Center, Tokyo Women's Medical University, Chiba, Japan \\ ${ }^{4}$ Department of Ophthalmology, Hachioji Medical Center, Tokyo Medical University, Tokyo, Japan
}

\begin{abstract}
:
Purpose:

The purpose of this study is to compare the aqueous humor level of Silent Information Regulator T1 (SIRT1) between patients with Age-related Macular Degeneration (AMD) and cataract patients.

Materials and Methods:

Aqueous humor level of SIRT1 was measured by enzyme-linked immunosorbent assay in 13 patients with wet-type AMD (n=13, AMD group) and 13 patients with cataracts (cataract group). In addition, the thickness of each retinal layer was determined by optical coherence tomography.

Results:

The aqueous humor level of SIRT1 was significantly lower in the AMD group than in the cataract group ( $\mathrm{p}=0.007)$. In the AMD group, the SIRT1 level was positively correlated with the thickness of the retinal ganglion cell layer $(\mathrm{r}=0.31)$ and the inner nuclear layer $(\mathrm{r}=0.76)$.

Conclusion:

The aqueous level of SIRT1 decreased as the ganglion cell layer and inner nuclear layer became thinner, suggesting that reduction of SIRT1 activity might be involved in the pathogenesis of this disease.
\end{abstract}

Keywords: Age-related macular degeneration, Aqueous humor, Silent information regulator T1, Ganglion, Cell, Cataract patients, Optic neuritis.

\begin{tabular}{|l|l|l|l|}
\hline Article History & Received: December 15, 2020 & Revised: June 1, 2021 & Accepted: June 13, 2021 \\
\hline
\end{tabular}

\section{INTRODUCTION}

It has been suggested that sirtuins are involved in aging [1, 2]. Sirtuin overexpression prolongs the lifespan of various organisms, whereas sirtuin deletion or mutation shortens survival [3 - 5]. In humans, a total of seven sirtuin homologues designated as Silent Information Regulator T1 (SIRT1) to SIRT7 have been identified [6,7].

SIRT1 deacetylates both histone and non-histone proteins to regulate gene transcription and protein function. It is an important anti-aging molecule with a role in preventing agerelated diseases induced by oxidative stress. Various studies have assessed the role of SIRT1 in the eye $[8,9]$, including its

* Address correspondence to this author at the Department of Ophthalmology, School of Medicine, Teikyo University, 2-11-1 Kaga, Itabashi-ku, Tokyo, 173-8605 Japan; Tel: +81-3-3964-1211, Fax: +81-3-3964-1402;

E-mail: mimurat-tky@umin.ac.jp relationship with cataract $[10,11]$, retinal degeneration [12], [13 - 19], optic neuritis [20], and uveitis [21]. We previously examined the relation between SIRT1 and age-related cataract, revealing that the aqueous humor level of SIRT1 was correlated with visual acuity and with the severity of nuclear cataract [22]. Such findings support the hypothesis that a decrease SIRT1 activity is involved in the initiation of agerelated ocular disease. For example, SIRT1 may have a role in the pathogenesis of Age-related Macular Degeneration (AMD). However, the possible role of SIRT1 in AMD has received little attention.

A literature search did not identify any reports correlating SIRT1 levels in aqueous with retinal degeneration in any animal model. However, Kilic et al . reported significant higher blood levels of SIRT1 in older people $(n=103)$ than younger people, including children $(n=120)$ and adults $(n=115)$ and a significant positive correlation between blood SIRT1 level and 
age in the overall studied population [23]. They concluded that SIRT1 level may be related to decreased antioxidant enzyme and oxidative stress-induced products in older patients [23, 24].

Prior studies have shown that aqueous humor levels of various cytokines are involved in the pathogenesis and pathology of AMD $[25,26]$. SIRT1 activation shows promise for the treatment of aging and age-related diseases. Furthermore, vitreous levels of cytokines are correlated with aqueous humor levels of cytokines, but not correlated with plasma levels of cytokines in patients with proliferative diabetic retinopathy [27]. Therefore, the measurement of aqueous SIRT1 may be effective in understanding the pathological condition of AMD.

Accordingly, the present study was performed to compare the aqueous humor level of SIRT1 between AMD patients and cataract subjects in order to assess whether the intraocular SIRT1 concentration is associated with the pathogenesis of AMD. We also compared the SIRT1 level in aqueous humor between patients with typical AMD (tAMD) and those with Polypoidal Choroidal Vasculopathy (PCV), and we assessed correlations between SIRT1 and the thickness of each retinal layer measured by optical coherence tomography.

\section{MATERIALS AND METHODS}

\subsection{Study Design}

This study was conducted according to the principles of the Declaration of Helsinki, and the Institutional Review Board of Tokyo Women's Medical University Hospital (Tokyo, Japan). Informed consent was obtained from each subject.

\subsection{Subjects}

Thirteen patients with typical wet-type AMD (AMD group; 13 eyes) and 13 patients with cataract (cataract group; 13 eyes) were enrolled when presented to hospitals associated with Tokyo Women's Medical University Medical Center East. The AMD group comprised 6 patients with tAMD and 7 patients with PCV as shown in Table 1.

Patients in the AMD group were scheduled to undergo intravitreal injection of aflibercept ( $2 \mathrm{mg} / 0.05 \mathrm{ml}$; VEGF TrapEye, Eylea ${ }^{\circledR}$, Regeneron Pharmaceuticals, Inc., Tarrytown, NY, USA and Bayer Health Care Pharmaceuticals, Berlin, Germany) [25] and aqueous humor was collected just before treatment. Criteria for intravitreal aflibercept were macular edema involving the fovea (retinal thickness $>300 \mu \mathrm{m}$ ) and Best-Corrected Visual Acuity (BCVA) $<20 / 30$.

\subsection{Optical Coherence Tomography and Measurement of Retinal Thickness}

The intraretinal surfaces of the Internal Limiting Membrane (ILM), Nerve Fiber Layer (NFL), Ganglion Cell Layer (GCL), Inner Plexiform Layer (IPL), Inner Nuclear Layer (INL), Outer Nuclear Layer (ONL), photoreceptor Inner Segment/Outer Segment junction (IS/OS junction) or Ellipsoid Zone (EZ), and Retinal Pigment Epithelium (RPE)- Bruch's Membrane (BM) complex (RPE/BM) were defined semiautomatically by using automated retinal segmentation software in the OCT unit (Retinascan RS-3000; NIDEK, Gamagori, Japan) [28 - 31].

Diagnosis of Pigment Epithelium Detachment (PED), Subretinal Fluid (SRF), Cystoid Macular Edema (CME), and Subretinal Hemorrhage (SRH) was confirmed by both OCT and fundoscopy. PED was defined as the separation of the RPE from BM, while SRF and Serous Retinal Detachment (SRD) was defined as an optically clear space between the neurosensory retina and the RPE [25, 32].

Table 1. Clinical profile of the subjects.

\begin{tabular}{|c|c|c|c|}
\hline- & $\begin{array}{c}\text { Cataract } \\
\text { Group }\end{array}$ & $\begin{array}{c}\text { AMD } \\
\text { Group }\end{array}$ & $\begin{array}{c}\text { P value } \\
\text { (Cataract } \text { vs. } \\
\text { AMD) }\end{array}$ \\
\hline Number of Eyes & 13 & 13 & - \\
\hline Age (years) & $75.5 \pm 7.4$ & $75.2 \pm 5.8$ & $* 0.733$ \\
\hline Gender (Female/ Male) & $4 / 9$ & $6 / 7$ & ${ }^{* *} 0.344$ \\
\hline Refraction (D) & $1.9 \pm 6.9$ & $0.1 \pm 2.3$ & ${ }^{*} 0.402$ \\
\hline LogMAR visual acuity & $0.7 \pm 0.4$ & $0.7 \pm 0.5$ & ${ }^{*} 0.920$ \\
\hline Systemic Diseases & & & \\
\hline Hypertension & $4(30.8 \%)$ & $7(53.8 \%)$ & $* * 0.214$ \\
\hline Diabetes & $5(38.5 \%)$ & $3(23.1 \%)$ & $* * 0.386$ \\
\hline Hyperlipidemia & $1(7.7 \%)$ & $2(15.4 \%)$ & $* * 0.500$ \\
\hline Coronary heart disease & $3(23.1 \%)$ & $1(7.7 \%)$ & $* * 0.297$ \\
\hline Renal disease & $0(0.0 \%)$ & $0(0.0 \%)$ & $* * \mathrm{NA}$ \\
\hline Liver disease & $0(0.0 \%)$ & $0(0.0 \%)$ & ${ }^{*} \mathrm{NA}$ \\
\hline Cerebrovascular disease & $2(15.4 \%)$ & $2(15.4 \%)$ & $* * 0.703$ \\
\hline Alcohol intake & $0(0.0 \%)$ & $1(7.7 \%)$ & $* * 0.500$ \\
\hline Smoking & $0(0.0 \%)$ & $5(38.5 \%)$ & $* * 0.020$ \\
\hline Ocular Diseases & & & \\
\hline IOL & $0(0.0 \%)$ & $5(38.5 \%)$ & $* * 0.020$ \\
\hline Glaucoma & $0(0.0 \%)$ & $0(0.0 \%)$ & $* * \mathrm{NA}$ \\
\hline Diabetic retinopathy & $0(0.0 \%)$ & $0(0.0 \%)$ & $* * \mathrm{NA}$ \\
\hline
\end{tabular}

AMD = Age-related Macular Degeneration, IOL= Intraocular Lens. NA= Not Applicable. *Unpaired Student's t-test. ${ }^{*}$ Chi-square test of independence or Fisher's exact probability test.

\subsection{Collection of Aqueous Humor}

In the patients with AMD, aqueous humor samples were collected when intravitreal injection of aflibercept was performed. Limbal paracentesis of the anterior chamber was done with a 30 -gauge needle attached to an insulin syringe to collect aqueous humor (mean volume: $0.2 \mathrm{~mL}$ ). The aqueous humor samples were transferred to sterile plastic tubes immediately after collection and were stored at $-20{ }^{\circ} \mathrm{C}$ until analysis. Aqueous samples were also collected from the 13 cataract patients by limbal paracentesis at the time of routine cataract surgery and were stored frozen at $-20^{\circ} \mathrm{C}$.

SIRT1 levels in aqueous humor were measured by Enzyme-Linked Immunosorbent Assay (ELISA) using a Sirtuin 1 (human intracellular) ELISA Kit (BioVision Inc., Milpitas, CA, USA) according to the manufacturer's instructions [22].

\subsection{Statistical Analysis}

The unpaired Student's $t$-test was used to compare mean values between the two groups, while frequencies were 
compared with the chi-square test or Fisher's exact test. Correlation coefficients were calculated by Pearson's correlation analysis. Factors associated with the SIRT1 level in aqueous humor were investigated by multivariate logistic regression analysis, with the following explanatory variables: patient and ocular factors as shown in Table 2. OCT parameters including the thickness of various retinal layers were also used for the explanatory variables of another analysis. The level of significance was set at $p<0.05$ in all analyses.

\section{RESULTS}

The mean age (mean $\pm \mathrm{SD}$ ) was $75.2 \pm 5.8$ years (range: 67-87 years) in the AMD group and $75.5 \pm 7.4$ years (range: 65-86 years) in the cataract group (Table 1). Background characteristics of the study population are presented in Table $\mathbf{1}$. Among the 13 patients (13 eyes) in the AMD group, 7 patients (53.8\%) had hypertension, 3 patients $(23.1 \%)$ had diabetes, 2 patients $(15.4 \%)$ had hyperlipidemia, one patient $(7.7 \%)$ had coronary heart disease, and 2 patients $(15.4 \%)$ had cerebrovascular disease. None of them had renal disease or liver disease. Smoking was defined as current smoking. There were current smokers $(n=5)$, former smoker $(n=0)$, and nonsmokers (never smoked) $(\mathrm{n}=8)$ in the AMD group and currently smoker $(\mathrm{n}=0)$, former smoker $(\mathrm{n}=0)$, and nonsmokers $(n=13)$ in the cataract group. There were no significant differences of demographic or clinical characteristics between the cataract group and the AMD group. None of the patients had glaucoma or diabetic retinopathy (Table 1). There are no differences in the patient characteristics between CNV and PCV group (smoking, $n=2$ and $n=3$; hypertension, $n=3$ and $\mathrm{n}=4$; and diabetes $\mathrm{n}=1$ and $\mathrm{n}=2$ ). All patients in the cataract group had nuclear cataract. In cataract group, the grades of cataract were grade 3-4 according to the Emery-Little classification of nuclear hardness. Therefore, there was little difference in cataract grade in the cataract group.

BCVA (mean \pm standard deviation in LogMar units) was $0.7 \pm 0.5$ in the AMD group and $0.7 \pm 0.4$ in the cataract group. The mean duration of AMD was $35.7 \pm 50.3$ months (range: 1 157 months). PED was observed in 8 eyes $(61.5 \%, 4$ with tAMD and 4 with $\mathrm{PCV}$, respectively), while SRD was identified in 9 eyes $(69.2 \%, 4$ and 5), CME was found in 4 eyes $(30.8 \%, 3$ and 1$)$, and subretinal hemorrhage (SRH) was detected in 2 eyes ( 1 and 1 ).

In the AMD group, the mean thickness of the ILMRPE/BM was $388 \pm 94 \mu \mathrm{m}$, while the thickness of the GCL was $70.8 \pm 46 \mu \mathrm{m}$, INL was $89 \pm 29 \mu \mathrm{m}$, ONL was $127 \pm 60$ $\mu \mathrm{m}$, and IS/OS-RPE/BM was $56 \pm 20 \mu \mathrm{m}$.

Fig. (1) compares the SIRT1 level in aqueous humor between the cataract (cataract) group and the AMD groups. The SIRT1 concentration was significantly lower in the AMD group than in the cataract group $(\mathrm{p}=0.007$, two-tailed unpaired Student's t-test) (Fig. 1).

Table 2. Correlations between SIRT1 and background factors in the AMD group $(n=13)$ with multivariate odds ratios and $\mathbf{9 5} \%$ confidence intervals.

\begin{tabular}{|c|c|c|c|c|c|}
\hline- & Correlation Analysis & & \multicolumn{3}{|c|}{ Multivariate Analysis } \\
\hline Variable & R Value & $P$ Value & OR & $(95 \% \mathrm{CI})$ & $P$ Value \\
\hline Patient Factors & - & - & - & - & \\
\hline Age (years) & 0.40 & 0.181 & 0.97 & $(0.91-1.04)$ & 0.313 \\
\hline Gender $($ Male $=0 /$ Female $=1)$ & -0.08 & 0.806 & 0.95 & $(0.47-1.91)$ & 0.829 \\
\hline Hypertension & 0.42 & 0.157 & 1.08 & $(0.70-1.67)$ & 0.611 \\
\hline Diabetes & 0.44 & 0.135 & 1.12 & $(0.61-2.08)$ & 0.585 \\
\hline Hyperlipidemia & 0.02 & 0.941 & 0.68 & $(0.23-2.07)$ & 0.355 \\
\hline Coronary heart disease & 0.67 & 0.012 & 2.21 & $(1.00-4.86)$ & 0.050 \\
\hline Cerebrovascular disease & 0.19 & 0.542 & 1.47 & $(0.56-3.83)$ & 0.292 \\
\hline Smoking & 0.22 & 0.478 & 2.49 & $(0.37-16.78)$ & 0.224 \\
\hline Alcohol intake & -0.08 & 0.806 & 0.84 & $(0.35-2.04)$ & 0.583 \\
\hline Ocular factors & - & - & - & - & - \\
\hline Eye $($ Right $=0 /$ Left $=1)$ & 0.10 & 0.747 & 1.42 & $(0.70-2.86)$ & 0.167 \\
\hline IOP (mmHg) & -0.01 & 0.979 & 1.00 & $(0.98-1.01)$ & 0.677 \\
\hline Refraction (diopters) & 0.36 & 0.226 & 0.83 & $(0.45-1.52)$ & 0.320 \\
\hline LogMAR & 0.02 & 0.940 & 1.60 & $(0.53-4.88)$ & 0.210 \\
\hline No PVD before treatment & -0.20 & 0.511 & 1.10 & $(0.20-5.98)$ & 0.831 \\
\hline Cataract surgery (cataract=0/IOL $=1$ ) & -0.40 & 0.177 & 0.89 & $(0.33-2.44)$ & 0.675 \\
\hline Absence of posterior capsule & -0.50 & 0.084 & 0.26 & $(0.00-22.99)$ & 0.327 \\
\hline Type of AMD (tAMD $=0 / \mathrm{PCV}=1)$ & 0.14 & 0.646 & 1.29 & $(0.35-4.74)$ & 0.486 \\
\hline Duration of AMD (months) & 0.30 & 0.319 & 1.00 & $(0.99-1.01)$ & 0.609 \\
\hline AMD in the other eye & -0.02 & 0.949 & 0.83 & $(0.42-1.65)$ & 0.370 \\
\hline
\end{tabular}




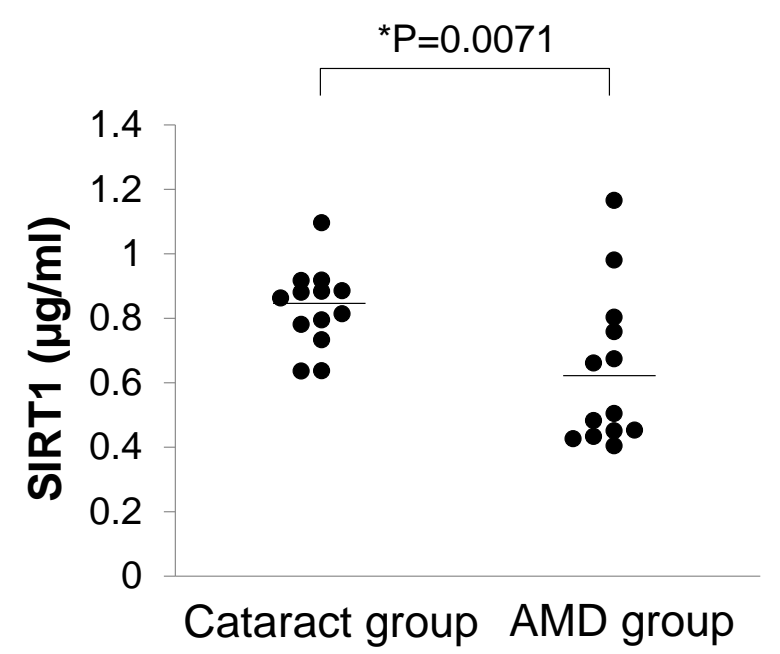

Fig. (1). SIRT1 levels in aqueous humor. Comparison between the cataract group and the AMD group. *Unpaired t-test.

We compared SIRT1 levels between the AMD group without cataract operation (non-IOL group, $n=8$ ) and the AMD group with cataract operation (IOL group, $n=5$ ). SIRT1 level tended to be higher in the non-IOL group $(0.70 \pm 0.24 \mu \mathrm{g} / \mathrm{ml})$ than in the IOL group $(0.51 \pm 0.15 \mu \mathrm{g} / \mathrm{ml})$, although no significant difference was found between the two groups $(\mathrm{p}=0.138)$.

We divided the AMD group into the non-smoking group $(n=8)$ and the smoking group $(n=5)$. SIRT1 levels were not significantly different between the non-smoking and smoking groups $(0.64 \pm 0.27 \mu \mathrm{g} / \mathrm{ml} v s .0 .61 \pm 0.14 \mu \mathrm{g} / \mathrm{ml}, \mathrm{p}=0.777)$.

Correlations were calculated to evaluate the relationships between SIRT1 and various factors. Table 2 shows correlations between the SIRT1 level and patient background factors in the

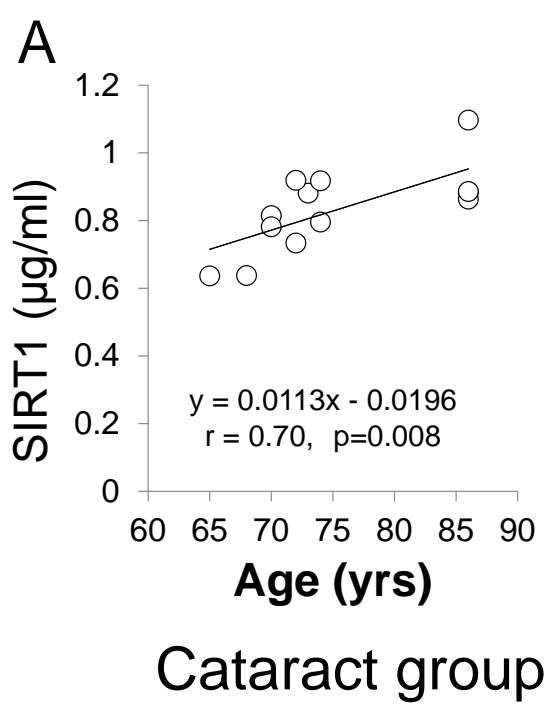

Fig. (2). Relationship between age and the SIRT1 level in aqueous humor. A, cataract group $(n=13)$. B, AMD group $(n=13)$.
AMD group, as well as the results of multivariate analysis. By Pearson's correlation analysis, SIRT1 was significantly correlated with coronary heart disease $(\mathrm{r}=0.67, \mathrm{p}=0.0122)$. Multivariate analysis also showed a significant association of coronary heart disease with the SIRT1 level (odds ratio [OR] = 2.21, $\mathrm{p}=0.050$ ). Fig. (2) displays the relationship between age and the SIRT1 level in aqueous humor. There was an agerelated increase of SIRT1 in the cataract group $(n=13, r=0.70$, $\mathrm{p}=0.008$ ) (Fig. 2A). However, there was no significant agerelated increase of SIRT1 in the AMD group ( $n=13, r=0.40$, $\mathrm{p}=0.181)$ (Fig. 2B) or across all subjects $(n=26, r=0.37$, $\mathrm{p}=0.060$ ). Additionally, we compared the background between the low SIRT1 group $(n=7)$ and the higher SIRT1 group $(n=8)$ according to the clusters of Fig. (2A). However, there were no significant differences in age $(76.1 \pm 3.2 v s .76 .8 \pm 3.4)$, gender (male/female $=4 / 4$ vs. $2 / 5)$, the presence of systemic diseases including hypertension $(3 / 8 v s .4 / 7)$, diabetes $(1 / 8$ vs. $2 / 7)$, hyperlipidemia (1/8vs. 1/7), coronary heart disease $(0 / 8 v s$. $1 / 7)$, cerebrovascular disease $(1 / 8$ vs. $1 / 7)$, smoking $(2 / 8 v s$. $3 / 7)$, alcohol intake (0/8 vs. 1/7), eye (right/left=4/4 vs. 5/2), presence of PVD (5/8 vs. 3/7), or history of cataract surgery (3/8 vs. 2/7) between the two groups.

Table 3 lists correlations between SIRT1 and OCT parameters in the AMD group. The SIRT1 concentration in aqueous humor was significantly correlated with the thickness of the INL ( $\mathrm{r}=0.76, \mathrm{p}=0.002$ ) (Table 3). According to multivariate analysis, the thickness of the ILM-RPE/BM $(\mathrm{OR}=0.41, \quad \mathrm{p}=0.006), \quad \mathrm{GCL} \quad(\mathrm{OR}=2.49, \quad \mathrm{p}=0.004), \quad \mathrm{INL}$ $(\mathrm{OR}=2.57, \mathrm{p}=0.001)$, and IS/OS-RPE/BM $(\mathrm{OR}=0.43, \mathrm{p}=0.018)$ were all factors associated with the SIRT1 concentration in AMD (Table 3). Fig. (3) shows the relationship between SIRT1 and three OCT parameters. The SIRT1 level was positively correlated with the thickness of the GCL (Fig. 3A) and the INL (Fig. 3B), while it was negatively correlated with the thickness of ONL (Fig. 3C). However, these correlations did not reach statistical significance. Fig. (3D) shows representative OCT images labeling each retinal layer.

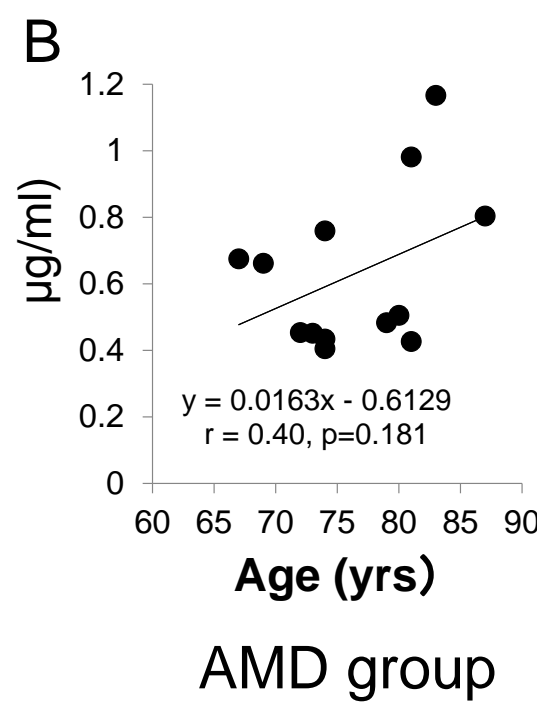



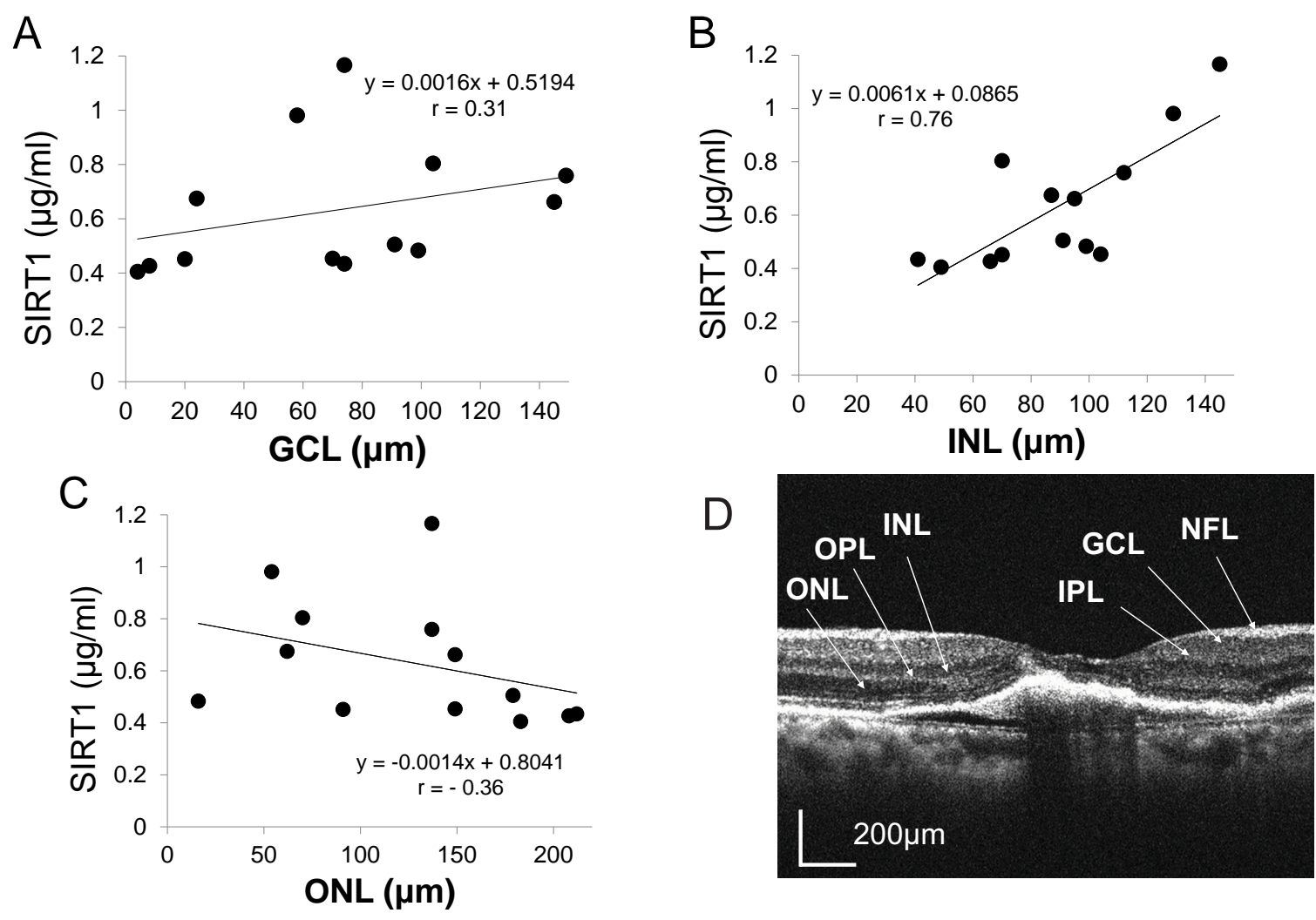

Fig. (3). Relationship between retinal thickness and SIRT1.

A, Ganglion Cell Layer (GCL). B, Inner Nuclear Layer (INL). C, Outer Nuclear Layer (ONL). D, OCT images of CNV showing each retinal layer. Nerve Fiber Layer (NFL). Inner Plexiform Layer (IPL). Outer Plexiform Layer (OPL).

Table 3. Correlations between SIRT1 and OCT parameters with multivariate odds ratios in the AMD group (n=13).

\begin{tabular}{|c|c|c|c|c|}
\hline \multirow[b]{2}{*}{ Variable } & \multirow{2}{*}{$\begin{array}{c}\text { Correlation Coefficient } \\
\mathbf{R}\end{array}$} & \multirow[b]{2}{*}{$P$ Value } & \multicolumn{2}{|c|}{ Multivariate Analysis } \\
\hline & & & OR & $P$ Value \\
\hline ILM-RPE/BM & -0.11 & 0.714 & 0.41 & 0.006 \\
\hline GCL & 0.31 & 0.297 & 2.49 & 0.004 \\
\hline INL & 0.76 & 0.002 & 2.57 & 0.001 \\
\hline ONL & -0.36 & 0.231 & 1.52 & 0.351 \\
\hline IS/OS-RPE/BM & -0.40 & 0.175 & 0.43 & 0.018 \\
\hline PED & 0.35 & 0.236 & 2.62 & $<0.001$ \\
\hline SRD & 0.32 & 0.286 & 0.40 & 0.004 \\
\hline
\end{tabular}

$\mathrm{R}$ value $=$ two-tailed Pearson's correlation coefficient calculated to assess associations between SIRT1 and each OCT parameter in the AMD group ( $\mathrm{N}=13$ ). ILM = Inner Limiting Membrane. RPE/BM = Retinal Pigment Epithelium-Bruch's Membrane. GCL $=$ Ganglion Cell Layer. INL $=$ Inner Nuclear Layer. ONL $=$ Outer Nuclear Layer. IS/OS $=$ junction of the inner and outer photoreceptor segments. PED = Pigment Epithelium Detachment. SRD = Serous Retinal Detachment. OR $=$ Odds Ratio.

Table 4. Correlations between SIRT1 and the presence of PED, SRF, CME, and SRH with multivariate odds ratios and 95\% confidence intervals $(n=13)$.

\begin{tabular}{|c|c|c|c|c|c|}
\hline Correlation Analysis & \multicolumn{4}{|c|}{ Multivariate Analysis } \\
\hline Variable & R-Value & $\boldsymbol{P}$-Value & OR & \multicolumn{1}{|c|}{ (95\% CI) } & $(0.72-1.63)$ \\
\hline PED & 0.26 & 0.386 & 1.08 & 0.664 \\
\hline SRF & 0.34 & 0.250 & 1.13 & $(0.73-1.74)$ & $(0.59-1.28)$ \\
\hline CME & -0.31 & 0.302 & 0.87 & 0.535 & 0.434 \\
\hline SRH & 0.00 & 0.988 & 0.88 & $0.563-1.46)$ \\
\hline
\end{tabular}

$\mathrm{R}$ value $=$ two-tailed Pearson's correlation coefficient. $\mathrm{OR}=$ Odds Ratio. $\mathrm{CI}=$ Confidence Interval. $\mathrm{PED}=$ Pigment Epithelium Detachment. $\mathrm{SRF}=\mathrm{Subretinal} \mathrm{Fluid.} \mathrm{CME}$ $=$ Cystoid Macular Edema. SRH $=$ Subretinal Hemorrhage . 
Table 5. Comparison of OCT parameters between patients with cataract and patients with IOL in the AMD group.

\begin{tabular}{|c|c|c|c|}
\hline & Patients with Cataract & Patients with IOL & $\begin{array}{c}\text { P-Value } \\
\text { (Cataract } \boldsymbol{v s .} \text { IOL) }\end{array}$ \\
\hline Number of Eyes & 8 & 5 & - \\
\hline ILM-RPE/BM $(\mu \mathrm{m})$ & $363 \pm 97$ & $430 \pm 73$ & $* 0.111$ \\
\hline GCL $(\mu \mathrm{m})$ & $80 \pm 46$ & $56 \pm 42$ & $* 0.204$ \\
\hline INL $(\mu \mathrm{m})$ & $105 \pm 22$ & $63 \pm 17$ & $* 0.003$ \\
\hline ONL $(\mu \mathrm{m})$ & $99 \pm 48$ & $170 \pm 52$ & $* 0.028$ \\
\hline IS/OS-RPE/BM $(\mu \mathrm{m})$ & $54 \pm 17$ & $60 \pm 24$ & $* .320$ \\
\hline Presence of PED & $4(50.0 \%)$ & $4(80.0 \%)$ & $* * 315$ \\
\hline Presence of SRF & $6(75.0 \%)$ & $3(60.0 \%)$ & $* * .510$ \\
\hline Presence of CME & $1(12.5 \%)$ & $3(60.0 \%)$ & $* * 0.119$ \\
\hline Presence of SRH & $1(12.5 \%)$ & $1(20.0 \%)$ & $* * 0.641$ \\
\hline
\end{tabular}

$\mathrm{OR}=$ Odds Ratio. $\mathrm{CI}=$ Confidence Interval. $\mathrm{PED}=$ Pigment Epithelium Detachment. SRF $=$ Subretinal Fluid. $\mathrm{CME}=\mathrm{Cystoid}$ Macular Edema. SRH $=$ Subretinal Hemorrhage.

*Unpaired Student's t-test. **Chi-square test of independence or Fisher's exact probability test.

The relations between SIRT1 and various features of AMD are displayed in Table 4 . There was no correlation between the SIRT1 level in aqueous humor and the presence of PED, SRF, CME, or SRH. Multivariate analysis also showed no association between SIRT1 and these retinal findings.

The OCT parameters were compared between patients with cataract $(n=8)$ and patients with IOL $(n=5)$ in the AMD group (Table 5). The thickness of INL was thicker in patients with cataract than in those with IOL $(105 \pm 22 \mu \mathrm{m} v s .63 \pm 17 \mu \mathrm{m}$, $\mathrm{p}=0.003)$. On the other hand, the thickness of ONL was thinner in patients with cataract than in those with IOL $(99 \pm 48 \mu \mathrm{m} v s$. $170 \pm 52 \mu \mathrm{m}, \mathrm{p}=0.003)$ (Table 5).

\section{DISCUSSION}

The present study demonstrated that the SIRT1 level in aqueous humor was significantly lower in patients with AMD than in cataract patients with cataract. SIRT1 was positively correlated with the thickness of the retinal GCL and INL, while it was negatively correlated with the ONL thickness. In addition, multivariate analysis revealed GCL and INL were predictors of the SIRT1 level. These findings suggest that SIRT1expression is decreased in AMD patients and that reduced SIRT1 activity is associated with the pathogenesis of AMD.

We observed a significant increase of the SIRT1 level in aqueous humor with age in the cataract group in this study (Fig. 2). Previous studies have also demonstrated that the SIRT1 protein level increases with age in rats [33], and humans $[23,34]$. On the other hand, the SIRT1 protein level was decreased in neurons from the hippocampus and cerebral cortex of aged rats $[35,36]$, and SIRT1 protein levels were decreased in cultured human vascular smooth muscle cells from elderly donors compared with cells from young donors [37]. Therefore, it is probable that the changes of SIRT1 during the ageing process differ among humans, animals, cultured cells, and different organs/tissues. In humans, SIRT1 protein has generally been found to increase with age [33, 34]. Recent studies have provided evidence that SIRT1 can prolong the lifespan or improve health [38, 39]. Kilik and associates reported a significant increase of the SIRT1 protein level in older people compared to younger people and a significant positive correlation of the SIRT1 level with age in older people ( $>55$ years), suggesting that humans regulate SIRT1 over their lifetime and that SIRT1 expression increases dramatically with age among the elderly [34]. Increased SIRT1 activity can protect cells and organs against oxidative stress. Two studies in rat models have shown that the SIRT1 protein level increases with aging, while reduced SIRT1 activity is related to oxidative damage $[33,40]$. Further, a decline of SIRT1 activity is related to a decline in nicotinamide adenine dinucleotide (NAD+) in aging mice [41]. NAD+ activates sirtuins, while nicotinamide (NAM) and the reduced form of NAD $+(\mathrm{NADH})$ are sirtuin inhibitors. SIRT1 catalyzes deacetylation and ADPribosylation reactions, with both enzymatic reactions involving cleavage of NAD + as a cofactor and production of NAM. The response of SIRT1 to oxidative stress is regulated by a NAD+dependent DNA repair enzyme, poly (ADP-ribose) polymerase-1 (PARP), and NAD + depletion by oxidative stress induces inflammation and the stress response [42]. Accordingly, the age-related increase of aqueous SIRT1 detected in the current study may suggest an increase of oxidative stress in the eye. Elevation of SIRT1 in older patients may be a compensatory mechanism for age- and oxidative stress-associated depletion of NAD + [34]. The reported positive association between SIRT1 and lipid metabolism also supports a compensatory role against the increase of oxidative stress with aging $[43,44]$.

SIRT1 level was slightly higher in the non-IOL group than in the IOL group. Previous report suggested that the SIRT1 levels in lens steadily decline during aging [11]. However, another study showed that SIRT1 expression was higher in patients older than 50 years with a diagnosis of cataract than in patients without cataract [10]. These results suggest that the lens epithelium may be a source of SIRT1 and SIRT1 may have a protective effect against senile cataract formation $[8,10$, $11]$.

Nicotine is known to reduce SIRT levels in other tissues $[45,46]$. However, our results showed no significant differences in the aqueous SIRT1 levels between smoking and non-smoking groups. There may have been no difference because the number of cases was small in our study. 
Interestingly, the SIRT1 level was lower in the AMD group than in the cataract group (Fig. 1). Both cataract and AMD become more frequent with increasing age, so our results may suggest that the anti-aging effect of SIRT1 is weaker in the retinas of AMD patients compared with cataract patients. Expression of both octamer binding transcription factor 4 (Oct4) and SIRT1 mRNA was reported to be significantly lower in donor eyes with AMD compared to donor eyes without AMD, which could be related to oxidative damage [18]. Peng and associates reported that overexpression of SIRT1 and Oct4 enhances antioxidant enzymatic activity in the retina, and activates reprogramming of RPE cells into retinal progenitor cells [18]. They also demonstrated in a rat model that Oct4 and SIRT1 gene transfer rescued retinal cell loss and improved electroretinographic responses after retinal damage by light-induced oxidative stress [18]. These findings suggest that a decline of SIRT1 activity may disrupt SIRT1-regulated retinal transcriptional activation and induce cellular senescence in AMD.

We evaluated the relationship between SIRT1 and OCT parameters in the AMD group (Table 3), revealing a significant positive correlation between the SIRT1 level and the INL thickness. The SIRT1 level was also positively correlated with the GCL thickness. Previous study reported the localization was seen in the retinal ONL, INL, and GCL in adult mouse eyes [14]. Several authors have already examined the thickness of each retinal layer in AMD by using OCT [47 - 49]. It was reported that the Ganglion Cell Complex (GCC) and the peripapillary retinal nerve fiber layer (RNFL) were thinner in AMD patients compared with normal controls [47]. Zucchiatti et al . also reported that the GCC and RNFL were thinner in AMD patients than in controls, becoming progressively thinner in the AMD group [48]. Thinning of the retinal cell layers including INL and GCL in AMD eyes would result in visual disturbance. Furthermore, multiple retinal cell layers were significantly thinner than normal in SIRT1-deficient adult mice, and the inner and outer nuclear layers showed disorganization [12]. Our findings support the hypothesis that the SIRT1 level progressively decreases as retinal cell layers (including GCL and INL) become thinner with the progression of AMD.

Next, the mechanism of retinal protection by SIRT1 needs to be considered. SIRT1 has a neuroprotective effect and the retina is part of the central nervous system despite its peripheral location. Because of its unique location in the eye, various factors such as smoking, UV radiation, aging, and oxidative stress can induce permanent damage to the retinal architecture [50]. SIRT1 is localized in most retinal layers, including the ONL, INL, GCL, and RPE, in the normal mouse retina [14], and recent evidence has supported a neuroprotective effect of SIRT1 in these retinal cell layers against retinal and optic nerve damage. Loss of E2 factor (the transcription factor for SIRT1), induces destabilization of p53 by negatively regulating SIRT1 deacetylase activity, resulting in hyperacetylation of p53 and increased p53-dependent activation of apoptosis in the mouse retina [15]. As mentioned above, SIRT1 gene transfer accompanied by Oct 4 rescues retinal cell loss and improves electroretinographic responses in rats with light-induced retinal injury [18]. Furthermore, up- regulation of SIRT1 by resveratrol protects cultured retinal cells from apoptotic death induced by anti-recoverin (Rec-1) and anti-enolase (Enol-1) antibodies [13]. Resveratrol is a natural polyphenol found in red grapes and red wine that enhances SIRT1 activity [51, 52]. Resveratrol also reduces activation of up-regulated retinal activator protein-1 by exposure to light and has a protective effect against phototoxic degeneration of the mouse retina [16]. Anti-aging therapy with resveratrol could also be an alternative treatment for retinal damage. Our present results showed that the SIRT1 level was most closely correlated with the INL thickness among the retinal cell layers. The INL contains the bodies of Müller glial cells, bipolar cells, amacrine cells, and horizontal cells. SIRT1 probably protects these retinal interneurons and retinal vascular endothelial cells against oxidative damage under physiological conditions, but age-related reduction of retinal cell NAD+ levels with the subsequent decline of SIRT1 activity may lead to vascular degeneration and neuronal cell death in the inner retina, resulting in progressive retinal degeneration $[8,9]$.

In our study, $38 \%$ of the AMD group were smokers. Smoking may affect the measurement of SIRT1. Nicotine generates peroxynitrite (ONOO-) which oxidizes amino acids and selectively inhibits SIRT $[45,46]$. Therefore, the low levels of SIRT1 observed in the AMD group may reflect the effect of smoking. However, the ELISA assay we used cannot recognize nitrated SIRT1. In the future, measurements of nitrated SIRT1 will be useful to prove the association between smoking and SIRT1 in patients with AMD.

The first limitation of this study was the small sample size, so our findings should be confirmed in a larger number of patients. Another limitation was that we did not examine the retinal localization of SIRT1 because we could not obtain fresh human retinal tissue from normal individuals. Third, the primary source of SIRT1 in aqueous humor has not been well understood. Fourth, the cataract group may not be a valid control. We could not collect aqueous humor from the normal subjects due to ethical issues. Therefore, we recruited cataract patients as controls instead of normal healthy individuals. In the current study, the SIRT1 level was significantly lower in AMD patients than in cataract patients. However, our previous study demonstrated that the aqueous humor level of SIRT1 was positively correlated with the progression of cataract in patients with age-related cataract. Therefore, a true control group without cataract may have lower levels of SIRT1 than the cataract group. At present, it is ethically impossible to collect the aqueous humor in patients without cataract. Therefore, the experimental design of this study could be fatally flawed due to the inability to compare SIRT1 levels in patients without cataract and patients with AMD.

In summary, this study confirmed an age-related increase in the aqueous humor level of the anti-ageing molecule SIRT1, indicating that SIRT1 may have a protective role during normal ocular aging. The SIRT1 level was significantly lower in AMD patients than in cataract patients. Furthermore, the SIRT1 level decreased in proportion to thinning of the inner retinal layers (GCL and INL).

The future direction of this study is to investigate the relationship between the progression of AMD and SIRT1. It 
will also be important to investigate whether the administration of SIRT1 can suppress the onset and progression of AMD in animal models.

\section{ETHICS APPROVAL AND CONSENT TO PARTICIPATE}

This trial was approved by our regional ethics committee (H24-2611) and was registered with the UMIN Clinical Trials Registry (UMIN000013684).

\section{HUMAN AND ANIMAL RIGHTS}

No Animals were used in this research. All human research procedures followed were in accordance with the ethical standards of the committee responsible for human experimentation (institutional and national), and with the Helsinki Declaration of 1975, as revised in 2013.

\section{CONSENT FOR PUBLICATION}

Informed consent was obtained from each subject.

\section{AVAILABILITY OF DATA AND MATERIALS}

Not applicable.

\section{FUNDING}

This work was supported by a Grant-in-Aid for Scientific Research from the Ministry of Education, Culture, Sports, Science and Technology of Japan [grant number 20H04347], and Foundation for the Study of Eye Diseases for the Elderly (Grant 2019).

\section{CONFLICT OF INTEREST}

The authors report no conflicts of interest. The authors alone are responsible for the content and for writing this paper.

\section{ACKNOWLEDGEMENTS}

Declared none.

\section{REFERENCES}

[1] Gotta M, Strahl-Bolsinger S, Renauld H, et al. Localization of Sir2p: the nucleolus as a compartment for silent information regulators. EMBO J 1997; 16(11): 3243-55.

[http://dx.doi.org/10.1093/emboj/16.11.3243] [PMID: 9214640]

[2] Guarente L, Kenyon C. Genetic pathways that regulate ageing in model organisms. Nature 2000; 408(6809): 255-62.

[http://dx.doi.org/10.1038/35041700] [PMID: 11089983]

[3] Kaeberlein M, McVey M, Guarente L. The SIR2/3/4 complex and SIR2 alone promote longevity in Saccharomyces cerevisiae by two different mechanisms. Genes Dev 1999; 13(19): 2570-80. [http://dx.doi.org/10.1101/gad.13.19.2570] [PMID: 10521401]

[4] Tissenbaum HA, Guarente L. Increased dosage of a sir-2 gene extends lifespan in Caenorhabditis elegans. Nature 2001; 410(6825): 227-30. [http://dx.doi.org/10.1038/35065638] [PMID: 11242085]

[5] Rogina B, Helfand SL. Sir2 mediates longevity in the fly through a pathway related to calorie restriction. Proc Natl Acad Sci USA 2004; 101(45): 15998-6003.

[http://dx.doi.org/10.1073/pnas.0404184101] [PMID: 15520384]

[6] Frye RA. Characterization of five human cDNAs with homology to the yeast SIR2 gene: Sir2-like proteins (sirtuins) metabolize NAD and may have protein ADP-ribosyltransferase activity. Biochem Biophys Res Commun 1999; 260(1): 273-9.

[http://dx.doi.org/10.1006/bbrc.1999.0897] [PMID: 10381378]

[7] Frye RA. Phylogenetic classification of prokaryotic and eukaryotic Sir2-like proteins. Biochem Biophys Res Commun 2000; 273(2):
793-8.

[http://dx.doi.org/10.1006/bbrc.2000.3000] [PMID: 10873683]

[8] Mimura T, Kaji Y, Noma H, Funatsu H, Okamoto S. The role of SIRT1 in ocular aging. Exp Eye Res 2013; 116: 17-26. [http://dx.doi.org/10.1016/j.exer.2013.07.017] [PMID: 23892278]

[9] Mimura T, Noma H, Funatsu H, Kondo A, Matsubara M. Retinal neuroprotective effect of sirtuins. JSM Ophthalmol 2014; 2(1): 1-4.

[10] Zheng T, Lu Y. Changes in SIRT1 expression and its downstream pathways in age-related cataract in humans. Curr Eye Res 2011; 36(5): 449-55.

[http://dx.doi.org/10.3109/02713683.2011.559301] [PMID: 21501079]

[11] Lin TJ, Peng CH, Chiou SH, et al. Severity of lens opacity, age, and correlation of the level of silent information regulator T1 expression in age-related cataract. J Cataract Refract Surg 2011; 37(7): 1270-4. [http://dx.doi.org/10.1016/j.jcrs.2011.02.027] [PMID: 21700104]

[12] Cheng HL, Mostoslavsky R, Saito S, et al. Developmental defects and p53 hyperacetylation in Sir2 homolog (SIRT1)-deficient mice. Proc Natl Acad Sci USA 2003; 100(19): 10794-9.

[http://dx.doi.org/10.1073/pnas.1934713100] [PMID: 12960381]

[13] Anekonda TS, Adamus G. Resveratrol prevents antibody-induced apoptotic death of retinal cells through upregulation of Sirt1 and Ku70. BMC Res Notes 2008; 1: 122

[http://dx.doi.org/10.1186/1756-0500-1-122] [PMID: 19046449]

[14] Jaliffa C, Ameqrane I, Dansault A, et al. Sirt1 involvement in rd10 mouse retinal degeneration. Invest Ophthalmol Vis Sci 2009; 50(8): 3562-72.

[http://dx.doi.org/10.1167/iovs.08-2817] [PMID: 19407027]

[15] Chen D, Pacal M, Wenzel P, Knoepfler PS, Leone G, Bremner R. Division and apoptosis of E2f-deficient retinal progenitors. Nature 2009; 462(7275): 925-9.

[http://dx.doi.org/10.1038/nature08544] [PMID: 20016601]

[16] Kubota S, Kurihara T, Ebinuma M, et al. Resveratrol prevents lightinduced retinal degeneration via suppressing activator protein-1 activation. Am J Pathol 2010; 177(4): 1725-31.

[http://dx.doi.org/10.2353/ajpath.2010.100098] [PMID: 20709795]

[17] Ozawa Y, Kubota S, Narimatsu T, et al. Retinal aging and sirtuins. Ophthalmic Res 2010; 44(3): 199-203.

[http://dx.doi.org/10.1159/000316484] [PMID: 20829644]

[18] Peng CH, Cherng JY, Chiou GY, et al. Delivery of Oct4 and SirT1 with cationic polyurethanes-short branch PEI to aged retinal pigment epithelium. Biomaterials 2011; 32(34): 9077-88.

[http://dx.doi.org/10.1016/j.biomaterials.2011.08.008] [PMID: 21890195]

[19] Geng Y, Wang J, Liang J, Xu C, Zhi Y. Expression of Sirt1 and Sirt2 in injured optic retina of calorie restricted rats. Yan Ke Xue Bao 2011; 26(4): 221-4.

[PMID: 22187307]

[20] Shindler KS, Ventura E, Rex TS, Elliott P, Rostami A. SIRT1 activation confers neuroprotection in experimental optic neuritis. Invest Ophthalmol Vis Sci 2007; 48(8): 3602-9. [http://dx.doi.org/10.1167/iovs.07-0131] [PMID: 17652729]

[21] Kubota S, Kurihara T, Mochimaru H, et al. Prevention of ocular inflammation in endotoxin-induced uveitis with resveratrol by inhibiting oxidative damage and nuclear factor-kappaB activation. Invest Ophthalmol Vis Sci 2009; 50(7): 3512-9.

[http://dx.doi.org/10.1167/iovs.08-2666] [PMID: 19279313]

[22] Kondo A, Goto M, Mimura T, Matsubara M. Silent information regulator $\mathrm{T} 1$ in aqueous humor of patients with cataract. Clin Ophthalmol 2016; 10: 307-12

[http://dx.doi.org/10.2147/OPTH.S100213] [PMID: 26929595]

[23] Kilic U, Gok O, Erenberk U, et al. A remarkable age-related increase in SIRT1 protein expression against oxidative stress in elderly: SIRT1 gene variants and longevity in human. PLoS One 2015; 10(3)e 0117954

[http://dx.doi.org/10.1371/journal.pone.0117954] [PMID: 25785999]

[24] Elibol B, Kilic U. High levels of SIRT1 expression as a protective mechanism against disease-related conditions. Front Endocrinol (Lausanne) 2018; 9: 614.

[http://dx.doi.org/10.3389/fendo.2018.00614] [PMID: 30374331]

[25] Rezar-Dreindl S, Sacu S, Eibenberger K, et al. The intraocular cytokine profile and therapeutic response in persistent neovascular age-related macular degeneration. Invest Ophthalmol Vis Sci 2016; 57(10): 4144-50.

[http://dx.doi.org/10.1167/iovs.16-19772] [PMID: 27537264]

[26] Sato T, Takeuchi M, Karasawa Y, Enoki T, Ito M. Intraocular inflammatory cytokines in patients with neovascular age-related macular degeneration before and after initiation of intravitreal 
injection of anti-VEGF inhibitor. Sci Rep 2018; 8(1): 1098. [http://dx.doi.org/10.1038/s41598-018-19594-6] [PMID: 29348424]

[27] Wu F, Phone A, Lamy R, et al. Correlation of aqueous, vitreous, and plasma cytokine levels in patients with proliferative diabetic retinopathy. Invest Ophthalmol Vis Sci 2020; 61(2): 26. [http://dx.doi.org/10.1167/iovs.61.2.26] [PMID: 32084272]

[28] Okuma H, Mimura T, Goto M, et al. Effect of aflibercept in patients with age-related macular degeneration. Int Ophthalmol 2016; 36(2): 159-69.

[http://dx.doi.org/10.1007/s10792-015-0089-z] [PMID: 26043678]

[29] Noma H, Funatsu H, Mimura T, Harino S, Hori S. Aqueous humor levels of vasoactive molecules correlate with vitreous levels and macular edema in central retinal vein occlusion. Eur J Ophthalmol 2010; 20(2): 402-9.

[http://dx.doi.org/10.1177/112067211002000222] [PMID: 19967679]

[30] Noma H, Mimura T, Yasuda K, Shimura M. Role of soluble vascular endothelial growth factor receptors-1 and -2 , their ligands, and other factors in branch retinal vein occlusion with macular edema. Invest Ophthalmol Vis Sci 2014; 55(6): 3878-85.

[http://dx.doi.org/10.1167/iovs.14-13961] [PMID: 24894397]

[31] Noma H, Mimura T, Yasuda K, Shimura M. Role of soluble vascular endothelial growth factor receptor signaling and other factors or cytokines in central retinal vein occlusion with macular edema. Invest Ophthalmol Vis Sci 2015; 56(2): 1122-8.

[http://dx.doi.org/10.1167/iovs.14-15789] [PMID: 25634982]

[32] Noma H, Mimura T, Eguchi S. Association of inflammatory factors with macular edema in branch retinal vein occlusion. JAMA Ophthalmol 2013; 131(2): 160-5.

[http://dx.doi.org/10.1001/2013.jamaophthalmol.228] [PMID: 23411880]

[33] Koltai E, Szabo Z, Atalay M, et al. Exercise alters SIRT1, SIRT6, NAD and NAMPT levels in skeletal muscle of aged rats. Mech Ageing Dev 2010; 131(1): 21-8. [http://dx.doi.org/10.1016/j.mad.2009.11.002] [PMID: 19913571]

[34] Quintas A, de Solís AJ, Díez-Guerra FJ, Carrascosa JM, Bogónez E. Age-associated decrease of SIRT1 expression in rat hippocampus: prevention by late onset caloric restriction. Exp Gerontol 2012; 47(2): 198-201.

[http://dx.doi.org/10.1016/j.exger.2011.11.010] [PMID: 22143179]

[35] Ng F, Wijaya L, Tang BL. SIRT1 in the brain-connections with agingassociated disorders and lifespan. Front Cell Neurosci 2015; 9: 64. [http://dx.doi.org/10.3389/fncel.2015.00064] [PMID: 25805970]

[36] Thompson AM, Wagner R, Rzucidlo EM. Age-related loss of SirT1 expression results in dysregulated human vascular smooth muscle cell function. Am J Physiol Heart Circ Physiol 2014; 307(4): H533-41. [http://dx.doi.org/10.1152/ajpheart.00871.2013] [PMID: 24973384]

[37] Satoh A, Brace CS, Rensing N, et al. Sirt1 extends life span and delays aging in mice through the regulation of $\mathrm{Nk} 2$ homeobox 1 in the DMH and LH. Cell Metab 2013; 18(3): 416-30.

[http://dx.doi.org/10.1016/j.cmet.2013.07.013] [PMID: 24011076]

[38] Mercken EM, Hu J, Krzysik-Walker S, et al. SIRT1 but not its increased expression is essential for lifespan extension in caloricrestricted mice. Aging Cell 2014; 13(1): 193-6.

[http://dx.doi.org/10.1111/acel.12151] [PMID: 23941528]

[39] Braidy N, Guillemin GJ, Mansour H, Chan-Ling T, Poljak A, Grant R. Age related changes in NAD + metabolism oxidative stress and Sirt1 activity in wistar rats. PLoS One 2011; 6(4)e19194

[http://dx.doi.org/10.1371/journal.pone.0019194] [PMID: 21541336]
[40] Ramsey KM, Mills KF, Satoh A, Imai S. Age-associated loss of Sirt1mediated enhancement of glucose-stimulated insulin secretion in beta cell-specific Sirt1-overexpressing (BESTO) mice. Aging Cell 2008; 7(1): 78-88.

[http://dx.doi.org/10.1111/j.1474-9726.2007.00355.x] [PMID: 18005249]

[41] Kim MY, Zhang T, Kraus WL. Poly(ADP-ribosyl)ation by PARP-1: 'PAR-laying' NAD+ into a nuclear signal. Genes Dev 2005; 19(17): 1951-67.

[http://dx.doi.org/10.1101/gad.1331805] [PMID: 16140981]

[42] Morena M, Cristol JP, Dantoine T, Carbonneau MA, Descomps B, Canaud B. Protective effects of high-density lipoprotein against oxidative stress are impaired in haemodialysis patients. Nephrol Dial Transplant 2000; 15(3): 389-95.

[http://dx.doi.org/10.1093/ndt/15.3.389] [PMID: 10692526]

[43] Zhu W, Chen S, Li Z, et al. Effects and mechanisms of resveratrol on the amelioration of oxidative stress and hepatic steatosis in KKAy mice. Nutr Metab (Lond) 2014; 11: 35.

[http://dx.doi.org/10.1186/1743-7075-11-35] [PMID: 25140191]

[44] Di Vincenzo S, Heijink IH, Noordhoek JA, et al. SIRT1/FoxO3 axis alteration leads to aberrant immune responses in bronchial epithelial cells. J Cell Mol Med 2018; 22(4): 2272-82.

[http://dx.doi.org/10.1111/jcmm.13509] [PMID: 29411515]

[45] Ding Y, Han Y, Lu Q, et al. Peroxynitrite-mediated SIRT (Sirtuin)-1 inactivation contributes to nicotine-induced arterial stiffness in mice. Arterioscler Thromb Vasc Biol 2019; 39(7): 1419-31.

[http://dx.doi.org/10.1161/ATVBAHA.118.312346] [PMID: 31092012]

[46] Rimayanti U, Kiuchi Y, Yamane K, et al. Inner retinal layer comparisons of eyes with exudative age-related macular degeneration and eyes with age-related macular degeneration and glaucoma. Graefes Arch Clin Exp Ophthalmol 2014; 252(4): 563-70. [http://dx.doi.org/10.1007/s00417-013-2496-z] [PMID: 24146272]

[47] Zucchiatti I, Parodi MB, Pierro L, et al. Macular ganglion cell complex and retinal nerve fiber layer comparison in different stages of age-related macular degeneration. Am J Ophthalmol 2015; 160(3): 602-607.e1.

[http://dx.doi.org/10.1016/j.ajo.2015.05.030] [PMID: 26052088]

[48] Camacho P, Dutra-Medeiros M, Páris L. Ganglion cell complex in early and intermediate age-related macular degeneration: Evidence by SD-OCT manual segmentation. Ophthalmologica 2017; 238(1-2): 31-43.

[http://dx.doi.org/10.1159/000468965] [PMID: 28505617]

[49] Fletcher AE. Free radicals, antioxidants and eye diseases: evidence from epidemiological studies on cataract and age-related macular degeneration. Ophthalmic Res 2010; 44(3): 191-8.

[http://dx.doi.org/10.1159/000316476] [PMID: 20829643]

[50] Howitz KT, Bitterman KJ, Cohen HY, et al. Small molecule activators of sirtuins extend Saccharomyces cerevisiae lifespan. Nature 2003; 425(6954): 191-6.

[http://dx.doi.org/10.1038/nature01960] [PMID: 12939617]

[51] Baur JA. Biochemical effects of SIRT1 activators. Biochim Biophys Acta $2010 ; 1804(8): 1626-34$

[http://dx.doi.org/10.1016/j.bbapap.2009.10.025] [PMID: 19897059]

[52] Haigis MC, Sinclair DA. Mammalian sirtuins: Biological insights and disease relevance. Annu Rev Pathol 2010; 5: 253-95.

[http://dx.doi.org/10.1146/annurev.pathol.4.110807.092250] [PMID: 20078221]

\section{2021 Mimura et al.}

This is an open access article distributed under the terms of the Creative Commons Attribution 4.0 International Public License (CC-BY 4.0), a copy of which is available at: https://creativecommons.org/licenses/by/4.0/legalcode. This license permits unrestricted use, distribution, and reproduction in any medium, provided the original author and source are credited. 